\title{
Golikov Theorem of Ideal Time-of-Flight Mass Analyzers
}

\author{
A.S. Berdnikov ${ }^{1}$ and K.V. Solovyev ${ }^{2}$ \\ 1. Institute for Analytical Instrumentation RAS, Rizskiy pr. 26, 190103 St.Petersburg, Russian \\ Federation \\ 2. Saint-Petersburg State Polytechnical University, Polytechnicheskaya ul. 29, 195251 St.Petersburg, \\ Russian Federation
}

\section{Introduction}

Charged Particle Optics contains a lot of specific conservation laws most of which are absent in classical (light) optics: conservation of energy, conservation of momentum, conservation of phase space volume, scaling relations for coordinates, energies and voltages, symplectic relations for aberration coefficients, symmetry relations for aberration coefficients, Scherzer and Yakushev inequalities for plane and axially symmetrical systems with straight axis, illuminated area identity for the rigidity dispersion in magnetic and electrostatic sector fields, etc. [1-3].

Among these statements (which restrict greatly the characteristics of the CPO systems which can be achieved by electric and magnetic fields) there is the estimation for the quality of TOF systems suggested by Yu. K. Golikov [4]. Namely, if there is the periodical multi-cascade TOF system with the time-of-flight data measured along the path of the particle $[5,6]$ there are two and only two possibilities:

a) there is at least one nonzero time-to-energy aberration coefficient of some order and in this case the TOF system demonstrates the saturation effect — its resolution power limits to a fixed finite value even if the number of the cascades limits to infinity;

b) all time-to-energy aberration coefficients of any order are exactly zero; hence this is an ideal TOF system where the resolution power limits to infinity with the increase of the number of the cascades.

The absence of all time-to-energy aberrations results to the conclusion that systems [7] are the only candidates to TOF systems without the saturation effect. Manual verification proves immediately that for [7] all time-of-flight aberrations are zero and hence [7] are the only systems with no saturation. Further we will call such $N$-cascade systems as the ideal TOF systems.

The original rough estimation [4] is analyzed here with better mathematical rigor hoping to find new classes of the ideal TOF systems. The basic theorem proposed by Yu. K. Golikov is proved here with greater degree of generality and new candidates to the ideal TOF systems are introduced.

\section{Problem specification}

Suppose there is a periodical multi-cascade TOF system like in ref. [5,6]. The resolution $R$ of a single cascade is defined by the expression

$$
R=\frac{m}{\Delta m}=\frac{1}{2} \frac{T}{\Delta T}=\frac{1}{2} \frac{T}{\Delta t+\delta T} \leq \frac{1}{2} \frac{T}{\delta T}
$$

where $R$ in the reference mass, $\Delta m$ is the minimal distance between two neighboring mass peaks which can be resolved, $T$ is the time of flight value, $\Delta T$ is the time peak width, $\Delta t$ is the initial time spread, $\delta T$ is the aberrational time spread due to deviations of the beam parameters with respect to energy, 
angles and coordinates [3]. The resolution $R_{N}$ of the $N$-cascade system is defined by the expression

$$
R_{N}=\frac{1}{2} \frac{T_{N}}{\Delta T_{N}}=\frac{1}{2} \frac{T \cdot N}{\Delta t+\delta T \cdot N}=\frac{1}{2} \frac{T}{(\Delta t / N)+\delta T} \leq \frac{1}{2} \frac{T}{\delta T}
$$

where $T_{N}=N \cdot T$ is the time of flight value for the $N$-cascade system and $\delta T_{N}=N \cdot \delta T$ is the accumulated aberrational time spread for the $N$-cascade system.

It can be seen from Eq. (2) that for $N \rightarrow \infty$ the resolution $R_{N}$ limits to a finite value $T / 2 \delta T$ while we could expect that it limits to infinity. The restriction of the initial beam spreads in energy, angles and coordinates can improve $\delta T$ and the same can be achieved by careful and highly professional optimization of the aberrational properties for a single cascade. However, even in this case the multiplying of the cascades can do only one thing: that is, to cancel the influence of the initial time spread of the beam. This well known effect is called the saturation of the resolution for the $N$-cascade TOF systems $[3,4]$.

However, the patent [7] introduces specific time-of-flight mass spectrometers called $Z^{2}$-TOF systems which are free from the saturation effect. That is, the greater is the number of passes the greater is the resolution which is strictly proportional to the number of passes (cascades) for these systems. The question is: how fundamental is the restriction in Eq. (2) and are there some other ideal TOF systems which do not suffer from it?

\section{Primary analysis of the problem and Golikov corollary}

The intrinsic mechanism of the limitation (2) is the assumption that the aberrational time spreads $\delta T_{k}$ gained by the particle when it passes through the cascade $k$ are added over the cascades. Strictly saying, this is not so. Namely, angles and coordinates of the particle at the exit from some cascade are not the same as that at the entry to this cascade. Since the output coordinates and angles are the input coordinates and angles for the next cascade, time of flight values $\delta T_{k}$ are different for different cascades and hence can compensate each other at least partially. That is, the cascades are identical electrically but fragments of the trajectory of the particle inside each cascade are different. Hence the values $\delta T_{k}$ of the aberrational time increments inside each cascade can be different as well and there is a chance that the rough estimation $\sum_{k=1, N} \delta T_{k} \sim N \cdot \delta T$ is not true any more for some specific cases.

However, the following simple consideration by $\mathrm{Yu}$. Golikov shows that in typical cases this should not happen. Let us consider the aberrational representation of the time of flight value $T(\delta E, \delta x, \delta a, \delta y, \delta b)$ inside a single cascade with respect to the variation of the initial energy $\delta E$, initial coordinates $\delta x, \delta y$ and initial angles $\delta a, \delta b$ :

$$
T(\delta E, \delta x, \delta a, \ldots) \approx T_{0}+\sum_{n \geq 1}\left[\sum_{k_{E}+k_{x}+k_{a}+k_{y}+k_{b}=n} C_{k_{E}, k_{x}, k_{a}, k_{y}, k_{b}}(\delta E / E)^{k_{E}}(\delta x)^{k_{x}}(\delta a)^{k_{a}}(\delta y)^{k_{y}}(\delta b)^{k_{b}}\right]
$$

Here $n$ is the order of the aberration coefficients, $C_{k_{E}, k_{x}, k_{a}, k_{y}, k_{b}}$ are the values of the aberration coefficients, $C_{k_{E}, k_{x}, k_{a}, k_{y}, k_{b}}(\delta E / E)^{k_{E}}(\delta x)^{k_{x}}(\delta a)^{k_{a}}(\delta y)^{k_{y}}(\delta b)^{k_{b}}$ is the aberrational magnitude corresponding to the aberration coefficient $C_{k_{E}, k_{x}, k_{a}, k_{y}, k_{b}}, k_{E}, k_{x}, k_{a}, k_{y}, k_{b}$ are the indices of the aberration coefficients and, 
simultaneously, the powers of individual terms inside the aberrational magnitudes.

While the mixed aberrational magnitudes $C_{k_{E}, k_{x}, k_{a}, k_{y}, k_{b}}(\delta E / E)^{k_{E}}(\delta x)^{k_{x}}(\delta a)^{k_{a}}(\delta y)^{k_{y}}(\delta b)^{k_{b}}$ can (in principle) compensate each other when the charge particle passes through different cascades the purely chromatic aberrational magnitudes $C_{k_{E}, 0,0,0,0} \cdot(\delta E / E)^{k_{E}}$ cannot do it: the energy deviation $\delta E$ and the basic energy $E$ are the same at the input and at the output of each cascade. Hence the magnitudes $C_{k_{E}, 0,0,0,0} \cdot(\delta E / E)^{k_{E}}$ should be always accumulated and the only chance not to have the saturation effect (2) is to have all chromatic aberrational coefficients $C_{k_{E}, 0,0,0,0}$ to be equal to zero exactly.

Fortunately this exotic case is not a rare beast in our optical jungles. That is, there are the onedimensional potential wells $U_{0}(z)$ where the charged particle oscillates with the same period regardless its initial kinetic energy [8-10]; the latter is equivalent to the requirement that all chromatic time aberrations $C_{k_{E}, 0,0,0,0}$ are exactly zeroes. All such potential well profiles can be represented in parametric form as

$$
z(\lambda)=z_{0}+L \cdot\left(\lambda+F\left(\lambda^{2}\right)\right) ; U_{0}(\lambda)=\frac{1}{2} U_{L} \lambda^{2}
$$

where $\lambda$ is the dimensionless parameter, $F(\xi)$ is an arbitrary smooth function, $z_{0}$ is the center of the potential well, $L$ is the scaling parameter along the axis $O Z, U_{L}$ is the scaling parameter of the potential (see [10], however, in [11] this formula is considered as an expression which is well known long before). The explicit dependence $U=U_{0}(z)$ can be derived by excluding the parameter $\lambda$ from the equations (4) but this can be finalized in analytical form only for just a few functions $F(\xi)$.

Suppose the charged particle makes isochronous oscillations along the axis $O Z$ in its vicinity. The initial conditions $x(0)=y(0)=0$ and $\dot{x}(0)=\dot{y}(0)=0$ are necessary to move the particle exactly along the axis $O Z$. The requirement $\forall k_{E}: C_{k_{E}, 0,0,0,0}=0$ means that the one-dimensional profile $U_{0}(z)=U(0,0, z)$ of electrostatic potential should be consistent with parameterization (4) for some properly selected function $F(\xi)$. The period of oscillations along the axis $O Z$ should be also independent from the variations of the initial coordinates $x$ and $y$ and the velocities $v_{x}$ and $v_{y}$. To get this feature it is necessary to represent the electric potential $U(x, y, z)$ as $U(x, y, z)=U_{2 D}(x, y)+U_{0}(z)$ where $U_{0}(z)$ is some isochronous potential distribution taken from Eq. (4) and $U_{2 D}(x, y)$ is an arbitrary function of two variables; otherwise the equation of motion $\ddot{z}=-\frac{q}{m} \frac{\partial U(x, y, z)}{\partial z}$ depends on $x, y$. It can be verified that for such potential the particle really performs strictly isochronous oscillations along the axis $O Z$ regardless its initial energy $E$, initial coordinates $x, y, z$ and initial angles projected to the axes $O X$ and $O Y$. Moreover, the requirement that the oscillations take place in the vicinity of the axis $O Z$ is not necessary any more for this potential; the motions along the axis $O Z$ and along the plane $O X Y$ are absolutely independent.

It can be verified easily that the only functions $U(x, y, z)$ which can be represented like 
$U(x, y, z)=U_{2 D}(x, y)+U_{0}(z)$ and which satisfy the Laplace equation $\frac{\partial^{2} U}{\partial x^{2}}+\frac{\partial^{2} U}{\partial y^{2}}+\frac{\partial^{2} U}{\partial z^{2}}=0$ are the functions

$$
U(x, y, z)=U_{2 D}(x, y)+\frac{1}{2} U_{L}\left(\frac{z-z_{0}}{L}\right)^{2}
$$

with $U_{2 D}(x, y)$ satisfying the $2 \mathrm{D}$ Poisson equation $\frac{\partial^{2} U_{2 D}}{\partial x^{2}}+\frac{\partial^{2} U_{2 D}}{\partial y^{2}}=-\frac{U_{L}}{L^{2}}$. Fortunately the functions $U_{0}(z)=\frac{1}{2} U_{L}\left(\frac{z-z_{0}}{L}\right)^{2}$ with $U_{L}>0$ are in agreement with the parameterization (4) if $F(\xi)=0$ and hence these functions are isochronous (well known result for harmonic oscillators [8,9]). Unfortunately this is the only case where we have isochronous oscillations along the axis $O Z$ and can satisfy the Laplace equation. The systems with such electrostatic potentials are called the $Z^{2}$-TOF systems in [7].

Thus there is the following Golikov's corollary:

1) only the systems which are chromatically ideal (isochronous) can demonstrate the ideal TOF properties;

2) $Z^{2}$-TOF systems are the only ones which are chromatically isochronous and simultaneously satisfy the Laplace equation;

3) it can be verified directly that for $Z^{2}$-TOF systems all time of flight aberrations are exactly zero, not only the chromatic ones;

4) hence $Z^{2}$-TOF systems are the only systems with ideal TOF properties.

\section{Clarification of Golikov corollary}

However, there is a small gap in these considerations. Namely, in Eq. (3) it is possible that not only the chromatic magnitudes are proportional to the number $N$ of the cascades but that some geometrical or mixed aberrational magnitudes $C_{k_{E}, k_{x}, k_{a}, k_{y}, k_{b}}(\delta E / E)^{k_{E}}(\delta x)^{k_{x}}(\delta a)^{k_{a}}(\delta y)^{k_{y}}(\delta b)^{k_{b}}$ are proportional to the number of the cascades as well. As a result the chromatic aberrations can be partially compensated by the geometrical and mixed aberrations (explicit formulas for first order $N$-cascade aberrations are in [12]) and the strict theory becomes more complicated.

To analyze this case the specific mathematical approach called in classical mechanics the "action and angle variables" can be used [13-15]. Namely, the Liouville's theorem states that for the analytical mechanical system which performs the motion in a restricted area of the phase space some specific substitution of variables always exists $[13,14]$. That is, there is always the transformation from the coordinates $x, y, z$ and momenta $p_{x}, p_{y}, p_{z}$ into the "angles" $\Theta\left(x, y, z, p_{x}, p_{y}, p_{z}\right)$, $\Phi\left(x, y, z, p_{x}, p_{y}, p_{z}\right), \Psi\left(x, y, z, p_{x}, p_{y}, p_{z}\right)$ and the "actions" $I\left(x, y, z, p_{x}, p_{y}, p_{z}\right), J\left(x, y, z, p_{x}, p_{y}, p_{z}\right)$, $K\left(x, y, z, p_{x}, p_{y}, p_{z}\right)$ which: a) transforms the Hamiltonian equations into new Hamiltonian equations, 
b) the Hamiltonian function $H\left(x, y, z, p_{x}, p_{y}, p_{z}\right)$ written in new variables depends on "actions" but not on "angles".

It can be proved that the analytical Hamiltonian system (in particular, the system which describes the motion of charged particles in electrostatic field) is the ideal TOF system if and only if it is represented in action and angle variables like

$$
H=C \cdot I+G(J, K)
$$

where $I$ is the leading action corresponding to the motion in isochronous direction, $J, K$ are the complementary actions, $C$ is the constant and $G(J, K)$ is an arbitrary function [4]. The relation (6) is very close to the relation (5) for the isochronous electrostatic potential for the $Z^{2}$-TOF systems; however, it is not exactly equivalent to it.

The requirement that some Hamiltonian system is the analytical one (that is, that two perturbed phase space trajectories are globally close to each other at infinite interval of time) is a very strong requirement [14]. However, typically we can assume that at least the linearized Hamiltonian system is analytical and hence that for the linearized Hamiltonian system the angle/action substitutions exist. It can be shown [4] that if nonlinear Hamiltonian system can be considered as a regular perturbation of its linearized version and if the linearized version is the ideal TOF system which is represented like in Eq. (6) then the source nonlinear Hamiltonian system is also free from the saturation effect of Eq. (2). This statement works in both directions: if the linearized Hamiltonian system is not the ideal one, cannot be represented as in Eq. (6) and hence suffer from the saturation effect of Eq. (2) then the source nonlinear Hamiltonian system suffer from the saturation effect of Eq. (2) as well; see [4] for the details. However, the criterion (6) does not work for exotic cases like bifurcations, non-linear Hamiltonian resonances, etc. - here the nonlinear Hamiltonian is not the regular perturbation of its linearized version. Fortunately the regularly perturbed systems cover $99 \%$ of the possible Hamiltonian systems.

Now we are ready to formulate the Golikov theorem as a set of strict and correct corollaries:

1) analytical Hamiltonian system is the ideal TOF system if and only if the Hamiltonian is represented as $H(I, J, K)=C \cdot I+G(J, K)$ in its angle/action coordinates (strict proof can be produced using the technique [4]);

2) nonlinear Hamiltonian system which is a regular perturbation of some analytical Hamiltonian system is the ideal TOF system if and only if the Hamiltonian of this analytical system is represented as $H(I, J, K)=C \cdot I+G(J, K)$ in its angle/action coordinates (this statement is the analog of the similar statement for the linearized Hamiltonian systems in [4] and the typical case of the analytical Hamiltonian system used for the comparison with the nonlinear Hamiltonian system is the linearized version of the nonlinear Hamiltonian system to be investigated);

3) $Z^{2}$-TOF systems [7] are the only regular electrostatic systems with the ideal TOF properties and with the straight line for time-of-flight oscillations;

4) the existence of ideal TOF systems with curvilinear and 3D motion which can be derived from the relation $H(I, J, K)=C \cdot I+G(J, K)$ for Hamiltonian functions or for the linearized Hamiltonian 
functions, should be investigated separately;

5) it is possible that some strongly non-analytical systems (bifurcations, resonances, etc.) with ideal TOF properties can exist in addition to analytical and nearly analytical TOF systems defined by the relation $H(I, J, K)=C \cdot I+G(J, K)$ for the Hamiltonian function (see [4] for the particular example).

\section{Conclusion}

The statement considered in this paper is mainly of academic interest and importance. Like other general conservation laws specific for Charged Particle Optics it rather describes what cannot be achieved by the CPO systems than suggests the concrete ways how to solve the problem under consideration. However, it seems that the basic relation $H(I, J, K)=C \cdot I+G(J, K)$ is not as "abstract" as it looks like from the first view. It can be used as the basis to derive new sets of the ideal Hamiltonian time of flight systems and some of them can be of real practical importance like it happens with [7]. However, the calculations necessary to do it are really huge and time consuming. This work is just started now but we hope that in some time there will be an effective and practical output from these academic investigations [16].

\section{References:}

[1] H Wollnik in "Optics of Charged Particles", (Academic Press), (1987).

[2] PW Hawkes and E Kasper in "Principles of Electron Optics, Vol. 1", (Academic Press), (1989).

[3] MI Yavor in "Optics of Charged Particle Analyzers", (Elsevier), (2009).

[4] AS Berdnikov, Nucl. Instrum. Meth. A 645 (2011), p. 197.

[5] H Wollnik and M Przewloka, Int. J. Mass Spectrom. 96 (1990), p. 267.

[6] M Yavor et al, Phys. Procedia 1 (2008), p. 391.

[7] Y Golikov et al, US patent no. 8237111 (2012).

[8] LD Landau and EM Lifshitz in "Mechanics, vol. 1 of A Course of Theoretical Physics", (Pergamon Press), (1969).

[9] M Urabe in "Nonlinear autonomous oscillations: analytical theory", (Academic Press), (1967).

[10] C Anton and JL Brun, Am. J. Phys. 76 (2008), p. 537.

[11] VV Amelkin and BS Kalitin in "Isochronous and pulse fluctuations of two-dimensional dynamical systems (in Russian)", (KomKniga), (2006).

[12] AS Berdnikov, Nucl. Instrum. Meth. A 645 (2011), p. 205.

[13] H Goldstein in "Classical Mechanics", (Addison-Wesley), (1980).

[14] VI Arnold in "Mathematical Methods of Classical Mechanics", (Springer), (1989).

[15] RZ Sagdeev, DA Usikov and GM Zaslavsky in "Nonlinear Physics: From the Pendulum to Turbulence and Chaos", (Harwood Academic Publishers), (1988).

[16] The authors acknowledge Dr. Sumio Kumashiro from Shimadzu Corp. for his initial decision to support the $Z^{2}$-TOF project and Dr. Mikhail Sudakov for his many useful discussions. 